\title{
Bloch Cavity Solitons in Nonlinear Resonators with Intracavity Photonic Crystals
}

\author{
Kestutis Staliunas, ${ }^{1}$ Oleg Egorov, ${ }^{2}$ Yuri S. Kivshar, ${ }^{3}$ and Falk Lederer ${ }^{2}$ \\ ${ }^{1}$ Institució Catalana de Recerca i Estudis Avançats (ICREA), Departament de Física i Enginyeria Nuclear, \\ Universitat Politècnica de Catalunya, Colom 11, 08222 Terrassa, Spain \\ ${ }^{2}$ Institute of Condensed Matter Theory and Solid State Optics, Friedrich-Schiller Universität Jena, \\ Max-Wien-Platz 1, 07743 Jena, Germany \\ ${ }^{3}$ Nonlinear Physics Centre, Research School of Physical Sciences and Engineering, Australian National University, \\ Canberra ACT 0200, Australia
}

(Received 8 February 2008; revised manuscript received 29 August 2008; published 8 October 2008)

\begin{abstract}
We predict a novel type of cavity solitons, Bloch cavity solitons, existing in nonlinear resonators with the refractive index modulated in both longitudinal and transverse directions and for both focusing (at normal diffraction) and defocusing (at anomalous diffraction) nonlinearities. We develop a modified mean-field theory and analyze the properties of these novel cavity solitons demonstrating, in particular, their substantial narrowing in the zero-diffraction regime.
\end{abstract}

Passive planar resonators with nonlinear optical response are known to exhibit many interesting effects such as optical bistability, modulational instability, and spatial pattern formation. They can also support spatially localized modes-cavity solitons (CS) [1-7]. When a transversally homogeneous resonator is illuminated by a plane wave (a holding beam), CSs appear as spatially localized domains in the reflected or transmitted fields, which are "switched up" (for bright CSs) or "switched down" (for dark CSs). The CSs are associated with the existence of two bistable states [1], and usually they appear in the neighborhood of modulation instability [2]. The properties of CSs have been studied both theoretically and experimentally during the last two decades [3-6]. Such CSs provide a specific example of more general type of dissipative solitons [7].

The crucial parameter for the CS existence is the net diffraction of the resonator: the bright CS exists for focusing nonlinearity only for normal (positive) diffraction. In mean-field models the CS size scales with the diffraction coefficient. Thus, potential applications of CSs in optical memory or routing devices depend much on diffraction, and they could be much improved by a proper diffraction management. One possibility for diffraction management is to employ structures with a spatially modulated refractive index (such as photonic crystals [8]) which, among other virtues, allow the modification of the diffraction properties of the propagating light beams. Diffractionless propagation of narrow beams in periodic photonic structures (PPS) has been demonstrated [9-11]. Modulation of the refractive index has been shown to modify the nonlinear light propagation, including the generation of different types of (conservative) spatial solitons for both focusing and defocusing nonlinearities [12-14], and also at the zero-diffraction point [15].

Also in dissipative systems the manipulation of diffraction by the periodic modulation of the refractive index has been already utilized. The modulation can modify and even inhibit modulational instability in nonlinear resonators [16]. Recently, the formation of discrete CSs [17], dissipative Bragg solitons [18,19] and multiband lattice-CSs [20] have been predicted. The special class of subdiffractive dissipative solitons-so-called midband solitons, has been shown to exist in lasers with a periodic lateral modulation of the refractive index [21] and in arrays of coupled Kerrnonlinear cavities [22]. So far, diffraction manipulation in dissipative systems was achieved by modulating the refractive index in the transverse direction only, and none of the previous studies addressed resonators with index modulation both in transversal and longitudinal dimensions [representing genuine two-dimensional (2D) intracavity PPS]. Only linear properties of such resonators have been recently considered so far [23].

In this Letter we provide the first study of nonlinear effects in a resonator filled with a medium modulated both in longitudinal and transverse directions [see Fig. 1(a)]. Note that we study the planar resonator filled by PPS, which is not to be confused by the microcavities appearing due to the defect in PPS [24]. Second, we merge two research fields: diffraction management of twodimensional Bloch waves and dissipative solitons in nonlinear resonators and demonstrate the existence of CSs in nonlinear resonators with 2D PPS terming these new solutions Bloch cavity solitons. Remarkably, we find that bright CSs exist for both focusing (in the normal diffraction regime) and defocusing (in the anomalous diffraction regime) nonlinearity being one consequence of the diffraction management. Next, we develop a modified mean-field theory describing the nonlinear response of a cavity with index modulation in both directions, and analyze the properties of CSs. This yields another practically important result of the diffraction management, namely, the formation of CSs near and at the zerodiffraction point. 

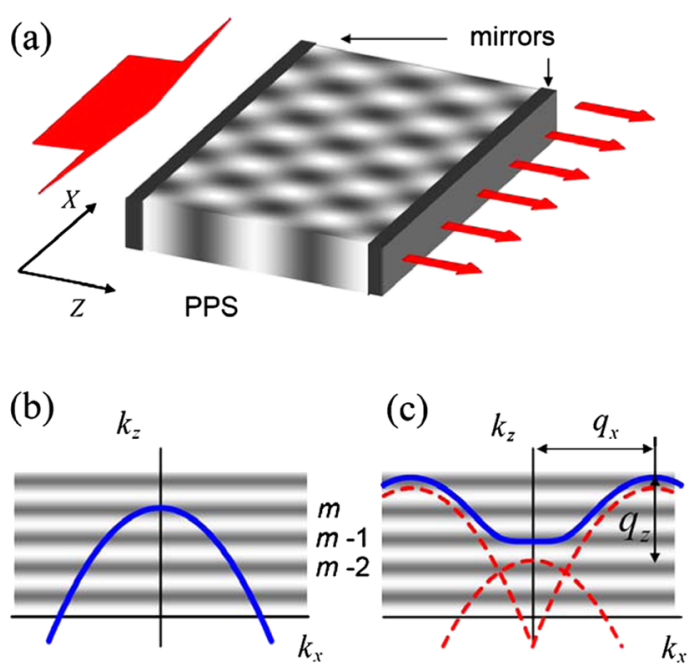

FIG. 1 (color online). (a) A nonlinear resonator with an intracavity two-dimensional photonic crystal. The dispersion relation of waves, here the isofrequency cureve, $k_{z}=f\left(k_{x}\right)$ for a homogeneous resonator (b) and for a 2D intracavity PPS (c) (the longitudinal mode structure of the cavity is depicted by stripes). The dashed lines in (c) indicate the dispersion curves in the limit of a vanishing modulation.

We consider a planar nonlinear resonator with the refractive index modulated in the longitudinal $(z)$ and one transverse $(x)$ direction, forming a two-dimensional periodic structure [Fig. 1(a)]. In the paraxial approximations, the traveling-wave model for this system can be written in the form,

$$
\begin{aligned}
{\left[i\left(v_{g}^{-1} \partial_{t} \pm \partial_{z}\right)\right.} & +\left(2 k_{0}\right)^{-1} \partial_{x x} \\
& \left.+V(x, z)+\gamma\left(\left|u^{ \pm}\right|^{2}+2\left|u^{\mp}\right|^{2}\right)\right] u^{ \pm}=0,
\end{aligned}
$$

where $u^{+}(x, z, t)$ and $u^{-}(x, z, t)$ are the amplitudes of the forward and backward propagating waves, respectively, $k_{0}=\omega_{0} n / c$ is the modulus of the wave vector, and $v_{g}$ is the group velocity. The term $V(x, z)=4 v \cos \left(q_{x} x\right) \times$ $\cos \left(q_{z} z\right)$ describes a periodical modulation of the refractive index in the resonator. We consider both focusing $(\gamma>0)$ and defocusing $(\gamma<0)$ Kerr nonlinearities. We account for the left $(z=0)$ and right $(z=l)$ resonator mirrors via the boundary conditions: $u^{+}(x, 0, t)=\rho \exp (i \phi) u^{-}(x, 0, t)+H$ and $u^{-}(x, l, t)=$ $\rho \exp (i \phi) u^{+}(x, l, t)$, where $\rho$ is the reflection coefficient of the mirrors (for simplicity, we assume identical mirrors) and $H$ is the amplitude of the holding beam. The net phase shift $\phi=\varphi_{0}+k_{0} l$ consists of the phase shift due the reflection at the mirrors $\varphi_{0}$ and due to propagation $k_{0} l$ [which is dropped from the propagation model (1) as a fast oscillating term].

First, we demonstrate the existence of stable CSs in the framework of the basic model (1). We find a stable bright $\mathrm{CS}$ in the homogeneous cavity for a focusing nonlinearity [Fig. 2(a)]. This CS is a non-mean-field analog of the wellstudied solution in the Lugiato-Lefever model [3]. Note
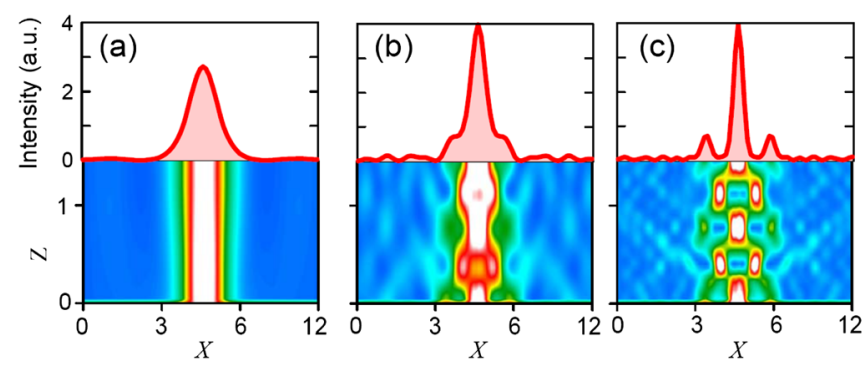

FIG. 2 (color online). Intensity profiles of the forward wave at the output of the resonator (upper plots), and the spatial distributions of the total (forward plus backward) intracavity field (bottom plots), calculated from the model (1) for (a) homogeneous cavity; (b) a weakly modulated cavity ( $v=$ 1) and a focusing nonlinearity $(\gamma=1)$; and (c) a strongly modulated cavity $(v=5)$ and a defocusing nonlinearity $(\gamma=$ $-1)$. Other parameters are: $l=1.5 l_{0}, q_{x}=5.45 \sqrt{2 k_{0} / l_{0}}, q_{z}=$ $16.76 l_{0}^{-1}, \rho=0.98, \phi=0.52 \pi$, and $H=1$, where $l_{0}$ is a normalization constant.

that CSs beyond the mean-field model have been so far obtained for a quadratic nonlinearity [25], but not for a cubic one. The intracavity index modulation changes the CS shape and modifies its existence domain. Nevertheless, stable bright CSs continue to exist for a focusing nonlinearity, at least for a weak modulation [see Fig. 2(b)]. The CSs now represent the envelopes of the Bloch modes of the PPS. When the index modulation becomes larger, the resonator diffraction changes its sign and becomes negative [10], and CSs ceases to exist for the focusing nonlinearity. In the inverted (anomalous) diffraction regime, stable bright CSs are possible only for a defocusing nonlinearity $(\gamma<0)$, as confirmed by our numerical studies [Fig. 2(c)].

The mean-field approach [3] is commonly used for describing homogeneous planar nonlinear cavities with a small round-trip field variation, if the operating frequency is close to the frequency of one longitudinal mode (for details, see Ref. [2]). Obviously, the conventional meanfield approach is not applicable in our case, since the modulation of the fields in the longitudinal direction of resonator is essential, due to the longitudinal component of intracavity modulation. To derive a modified mean-field model, first we expand the intracavity field into a set of spatial harmonics with the common transverse wave vector $k_{x}$. Figure 1 illustrates the main idea of our derivation where the isofrequency curve, which is the linear dispersion relation $k_{z}=f\left(k_{x}\right)$ at fixed frequency, is displayed without [Fig. 1(b)] and with [Fig. 1(c)] index modulation. In the homogeneous case, the paraxial approximation results in a parabolic dispersion curve $k_{z}=k_{0}-\left(2 k_{0}\right)^{-1} k_{x}^{2}$. The index modulation creates a set of parabolas, all shifted with respect to the basic one by integers of the wave vectors of the index modulation $\vec{q}=\left(m q_{x}, p q_{z}\right): k_{z, m, p}=$ $k_{0}-\left(2 k_{0}\right)^{-1}\left(k_{x}+m q_{x}\right)^{2}+p q_{z}$. We expand both (forward and backward) intracavity fields into a set of the plane waves: 


$$
\begin{aligned}
& u^{+}(x, z, t)=\sum_{m} \sum_{p} a_{m, p}(X, t) e^{i\left(m q_{x} x-p q_{z} z\right)}, \\
& u^{-}(x, z, t)=\sum_{m} \sum_{p} b_{m, p}(X, t) e^{i\left(m q_{x} x+p q_{z} z\right)},
\end{aligned}
$$

where the expansion coefficients depend on the large-scale transverse coordinate $X$ and time $t$. The index modulation introduces the coupling between the harmonics, which lifts the degeneracy at the intersections of the dispersion curves [Fig. 1(c)]. The presence of the resonator, in addition, imposes the resonance conditions on the longitudinal component of the Bloch vectors. We keep only three modes for each of the forward and backward propagating waves which suffices, as proven in Ref. [10], all relevant effects occur around the three intersections of the parabolas, as shown in Fig. 1(c). We apply then the mean-field approach separately for each harmonic. The resonator mirrors couple the corresponding forward and backward propagating harmonics. Inserting the expansion (2) into the round-trip model (1) and collecting the terms at the same harmonics, we obtain the mean-field equations,

$$
\begin{gathered}
\left(i \partial_{T}+D \partial_{X X}+i+\Delta\right) a_{0}+M\left(a_{+1}+a_{-1}\right) \\
+\delta a_{0}\left(3\left|a_{0}\right|^{2}+4\left|a_{+1}\right|^{2}+4\left|a_{-1}\right|^{2}\right)=E, \\
\left(i \partial_{T}+D \partial_{X X} \pm i P \partial_{X}+i+\Delta+\Omega\right) a_{ \pm 1} \\
+M a_{0}+\delta a_{ \pm 1}\left(4\left|a_{0}\right|^{2}+3\left|a_{ \pm 1}\right|^{2}+4\left|a_{\mp 1}\right|^{2}\right)=0,
\end{gathered}
$$

where $D=l / k_{0}\left(1-\rho^{2}\right)$ stands for the effective diffraction coefficient, and $\delta=2 l \gamma / 3\left(1-\rho^{2}\right)$ characterizes the strength of the cubic nonlinearity. $E=H /\left(1-\rho^{2}\right)$ is the normalized intensity of the holding beam. The time is normalized to the photon lifetime $\tau_{\mathrm{ph}}=2 l / v_{g}\left(1-\rho^{2}\right)$. The detunings of the wave components have the common part $\Delta=2 \ln \left(\omega_{0}-\omega_{m}\right) / c\left(1-\rho^{2}\right)$, which is related to the difference of the frequency of the holding beam $\omega_{0}$ and the frequency of the $m$ th longitudinal mode of the resonator $\omega_{m}$. The drift terms $P=2 l q /\left(1-\rho^{2}\right)$ and an additional detuning $\Omega=2 l\left[\left(2 k_{0}\right)^{-1} q_{x}^{2}-q_{z}\right] /\left(1-\rho^{2}\right)$ appear for the sideband components $a_{ \pm 1}$. The modulation parameter $M=2 l v /\left(1-\rho^{2}\right)$ mediates the coupling between the spatial harmonics. The derivation of the model (3) requires the assumption of large-scale variations of the fields compared with the scale of periodicity. The index modulation is considered to be weak, specifically the parameter $M / \Omega$ is considered to be small, which, expressed in initial variables read: $\quad \nu /\left|\left(2 k_{0}\right)^{-1} q_{x}^{2}-q_{z}\right| \ll 1$. The nonlinearity in Eqs. (3) is obtained by neglecting the higher harmonics, i.e., assuming that the fields are weak, which is ensured by the above "weak scattering" condition $|M / \Omega| \ll 1$.

The results of our numerical studies of Eqs. (3) are summarized in Figs. 3 and 4. We notice that without modulation (at $M=0$ ) the model (3) coincides with the Lugiato-Lefever model [3]. The existence of bright Bloch CSs for the focusing case $(\delta>0)$ and negative cavity detuning $[\Delta<0$, Figs. 3(a) and 3(d) $]$ is then evident. The periodic modulation transforms the intracavity waves into the envelopes of the Bloch modes. We calculate the position of the resonance in the linear limit in the term of the "conventional" cavity detuning $\Delta$ [see Figs. 3(a)-3(c)]. Therefore, we introduce the effective cavity detuning $\Delta^{\prime}$ which accounts for a shift to the nearest resonance position in the presence of the PC. Increasing the refractive index modulation, the conventional CSs transform into Bloch CSs and become narrower with an increasing modulation amplitude [see Figs. 3(b) and 3(e)]. We note that the width of the conventional CS depends drastically on the effective cavity detuning; i.e., the CS is broader for a smaller detuning. Therefore, we depict the dependence of the CS width (FWHM) on the modulation parameter $M$, keeping the effective cavity detuning $\Delta^{\prime}$ fixed (Fig. 4). The Bloch CS width attains a minimum shortly before the zerodiffraction point, which in our case appears at $M=700$. At close vicinity of the zero diffraction, the solitons cease to exist. The whole picture changes qualitatively beyond the zero-diffraction point, where the effective diffraction changes its sign [as evident from the dispersion curves in Figs. 3(b) and 3(c)]. Therefore, bright CSs cease to exist for the focusing nonlinearity just before the zerodiffraction point; however, they reappear for the defocusing nonlinearity and positive effective cavity detuning [see Figs. 3(c) and 3(f)]. The substantial reduction of the Bloch CS width by a factor of 2.5 or even more can be seen in Figs. 3(e) and 3(f). Because of the resonance between CS and linear modes the CS tails are modulated on the large spatial scale [see Fig. 3(e)] [the CS line "crosses" the
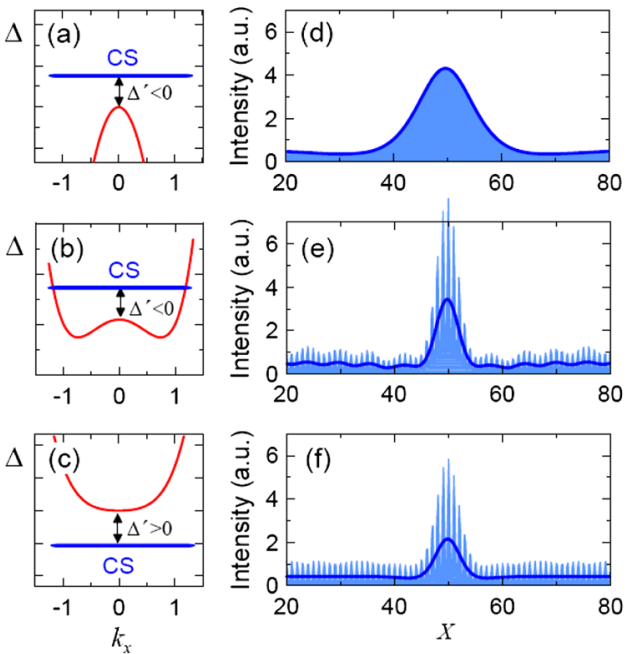

FIG. 3 (color online). (a)-(c) Dispersion for waves in resonance, and (d)-(f) intensity profiles of the corresponding CSs for different values of the modulation parameter $(M)$. The profiles are plotted for a CS in a homogeneous cavity (a),(d) and for Bloch CSs in a cavity with PPS $(M=600)$ for a focusing nonlinearity $\delta=1, \Delta^{\prime}=-2(\mathrm{~b}, \mathrm{e})$; and a cavity with PPS $(M=$ 800) for a defocusing nonlinearity $\delta=-1, \Delta^{\prime}=2$ (c),(f); Thick lines correspond to the central spatial harmonic $\left(\left|a_{0}\right|^{2}\right)$ and the shaded profiles result from the superposition of all three modes. Holding beam amplitude $E=1.37$. Other parameters are: $D=100, P=1257, \Omega=-2000$. 


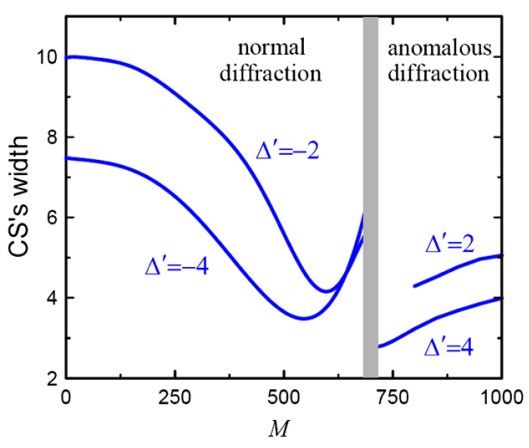

FIG. 4 (color online). The width of Bloch CSs vs the modulation parameter $M$ for different effective detunings $\Delta^{\prime}$. The vertical gray stripe marks the zero-diffraction regime. The left side of the zero-diffraction regime corresponds to normal diffraction and focusing nonlinearity: $\delta=1, E=1.37$ for $\Delta^{\prime}=$ -2 and $E=2.3$ for $\Delta^{\prime}=-4$. The right side is the case of anomalous (inverted) diffraction and defocusing nonlinearity: $\delta=-1, E=1.37$ for $\Delta^{\prime}=2$, and $E=2.3$ for $\Delta^{\prime}=4$.

linear dispersion curve in Fig. 3(b)]. We note, that the solitons of the mean-field limit (Figs. 3 and 4) continue to exist under conditions where the mean-field conditions are no more applicable (Fig. 2), which justifies their robustness with respect the non-mean-field generalizations.

Finally, we make a further simplification of the model (3) in order to interpret the rescaling of the effective diffraction in the system. As the system is dissipative, we can perform an adiabatic elimination of the weak sideband components from the second equation (3), considering that they are enslaved by the central component: $a_{ \pm 1} \approx$ $-\left(D \partial_{X X} \pm i P \partial_{X}+i+\Delta+\Omega\right)^{-1} M a_{0}$. Here we invert the evolution operator (including its differential part), which is mathematically correct since the spectrum of the inverted operator is not singular (due to the presence of losses). We consider a weak nonlinearity and, assuming, $\Omega \gg D \partial_{X X}$ and $P \partial_{X} \gg \Delta, 1$, expand above expression for $a_{ \pm}$into a series, $a_{ \pm 1}=M\left(-\Omega^{-1}+D \Omega^{-2} \partial_{X X} \pm\right.$ $\left.i P \Omega^{-2} \partial_{X}+P^{2} \Omega^{-3} \partial_{X X}+\ldots\right) a_{0}$. Inserting $a_{ \pm 1}$ into the first of Eq. (3) we obtain

$$
\left(i \partial_{T}+D_{1} \partial_{X X}+i+\Delta_{1}\right) a_{0}+\delta_{1} a_{0}\left|a_{0}\right|^{2}=E,
$$

which is the conventional model for the Kerr-nonlinear resonator [3]. In Eq. (4) the effective coefficients $D_{1}, \Delta_{1}$, and $\delta_{1}$ become modified with respect to the original coefficients $D, \Delta$, and $\delta$ due to the refractive index modulation. The most relevant is the modification of diffraction: $D_{1}=D\left[1+2 M^{2} / \Omega^{2}+2 M^{2} P^{2} /\left(\Omega^{3} D\right)\right]$. As $\Omega<0$, the last term in diffraction describes the basic phenomena analyzed above, namely, reduction, disappearance, and inversion of diffraction, and also allows us to evaluate the point where diffraction is arrested. The latter analytic evaluation corresponds well to the value calculated numerically from the traveling-wave model (1) as well as from the modified mean-field model (3). Thus the essential features of Bloch CSs can be understood from Eq. (4), namely, that soliton formation which is an interplay be- tween linear band nonlinear effects can be essentially controlled by the control of diffraction of Bloch waves.

In conclusion, we have predicted a novel type of cavity solitons in nonlinear resonators with a genuine twodimensional periodic modulation of the refractive index, with index modulated both in longitudinal and transverse directions.

This work was supported by Ministerio de Educación y Ciencia (Spain), European FEDER (FIS2005-07931-C0303), the Deutsche Forschungsgemeinschaft (research unit 532), Acciones Integradas Hispano Alemanas (HA-20060111), and the Australian Research Council.

[1] N. N. Rosanov, Spatial Hysteresis and Optical Patterns (Springer, Berlin, 2002)

[2] U. Peschel et al., IEEE J. Quantum Electron. 39, 51 (2003); D. Michaelis et al., IEEE J. Quantum Electron. 39, 255 (2003).

[3] L. A. Lugiato and R. Lefever, Phys. Rev. Lett. 58, 2209 (1987).

[4] M. Brambilla et al., Phys. Rev. Lett. 79, 2042 (1997); D. Michaelis et al., Phys. Rev. A 56, R3366 (1997).

[5] V. B. Taranenko et al., Phys. Rev. Lett. 81, 2236 (1998).

[6] V. B. Taranenko et al., Phys. Rev. A 61, 063818 (2000); S. Barland et al., Nature (London) 419, 699 (2002).

[7] Dissipative Solitons, edited by N. Akhmediev and A. Ankiewicz (Springer, Berlin, 2005).

[8] See, e.g., the review papers D. N. Christodoulides et al., Nature (London) 424, 817 (2003); D. Neshev et al., J. Nonlinear Opt. Phys. Mater. 16, 1 (2007), and references therein.

[9] H. Kosaka et al., Appl. Phys. Lett. 74, 1212 (1999).

[10] K. Staliunas and R. Herrero, Phys. Rev. E 73, 016601 (2006).

[11] R. Iliew et al., Appl. Phys. Lett. 85, 5854 (2004).

[12] Yu. S. Kivshar and G. P. Agrawal, Optical Solitons: From Waveguides to Photonic Crystals (Academic, San Diego, 2003).

[13] A. A. Sukhorukov and Yu. S. Kivshar, Phys. Rev. Lett. 87, 083901 (2001).

[14] D. Neshev et al., Phys. Rev. Lett. 93, 083905 (2004).

[15] K. Staliunas et al., Phys. Rev. E 73, 065603(R) (2006).

[16] D. Gomila et al., Phys. Rev. Lett. 92, 253904 (2004); D. Gomila and G. L. Oppo, Phys. Rev. E 72, 016614 (2005).

[17] U. Peschel et al., Opt. Lett. 29, 1909 (2004); O. A. Egorov et al., Opt. Express 15, 4149 (2007).

[18] A. G. Vladimirov et al., Opt. Express 14, 1 (2006).

[19] A. V. Yulin et al., Opt. Express 13, 3529 (2005).

[20] O. A. Egorov and F. Lederer, Phys. Rev. A 76, 053816 (2007).

[21] K. Staliunas, Phys. Rev. Lett. 91, 053901 (2003); K. Staliunas, Phys. Rev. E 70, 016602 (2004).

[22] O. Egorov et al., Opt. Lett. 32, 2106 (2007).

[23] K. Staliunas et al., Phys. Rev. A 76, 051803(R) (2007).

[24] M. Notomi et al., Opt. Express 13, 2678 (2005); M. Notomi et al., Opt. Express 15, 17458 (2007); Y. Tanaka et al., Nature Mater. 6, 862 (2007).

[25] M. Tlidi et al., Phys. Rev. A 61, 043806 (2000). 